\title{
Nitrosativer Stress im Gehirn - pathogene Auswirkung des Mobilfunks?
}

\author{
Jürgen Aschoff
}

\section{Schlüsselwörter}

Mobilfunk, Stickoxid (NO), nitrosativer Stress, Alzheimer, psychische Erkrankungen

\section{Zusammenfassung}

Stickoxid erfüllt verschiedene physiologische Aufgaben im Körper, z. B. im Immunsystem, im Gefäßsystem und bei der Steuerung des programmierten Zelltods. Zu große Mengen Stickoxid (nitrosativer Stress) führen jedoch zu Schäden im Organismus. Aus epidemiologischen Daten sowie aus dem Anstieg der Stickoxidexhalation unter Mobilfunkeinfluss lässt sich ein Zusammenhang zwischen der Zunahme des Mobilfunks und der steigenden Prävalenz psychischer Erkrankungen ableiten.

Die Zunahme des Mobilfunks wird in der Öffentlichkeit kontrovers diskutiert. Aus ärztlicher Sicht ist festzustellen, dass es keine ernstzunehmende Untersuchung zur Technikfolgenabschätzung gegeben hat, die insbesondere die Wirkung auf kranke und gesunde Menschen untersucht. Einziger betrachteter Parameter der Industrie und Aufsichtsbehörden der Politik ist die thermische Wirkung der Handystrahlung. Überspitzt gesagt wurde untersucht, ob eine Gewebeerwärmung - ähnlich dem Fleisch in der Mikrowelle - zu erwarten ist. Allein durch die geringe Abstrahlleistung war vorher klar, dass ein Erwärmungseffekt nicht auftreten wird.

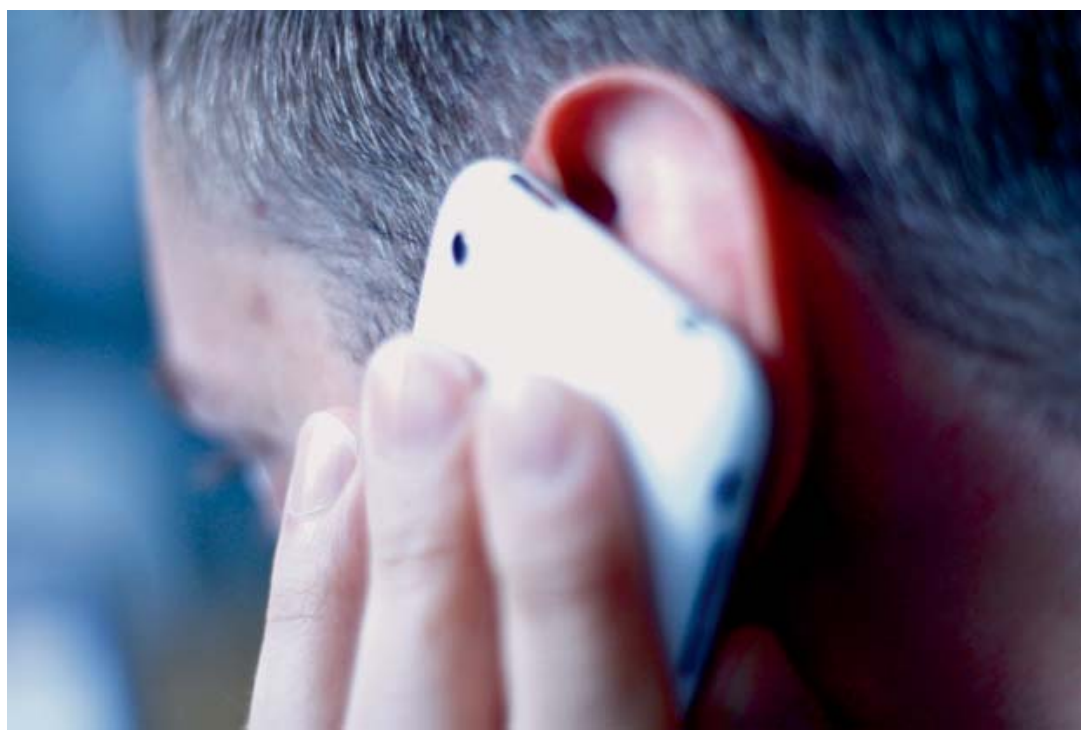

Ob häufiges Telefonieren mit Mobilfunkgeräten der Gesundheit schadet, ist nicht eindeutig erwiesen. Es mehren sich aber Hinweise auf einen Zusammenhang mit einem Anstieg psychischer Erkrankungen. (c) Robert Müller/pixelio.de.

\section{Effekte der Mobilfunkstrahlung}

Biologische Organismen haben komplizierte regulative, teils auch chaotische und überschießende Regelkreise mit interindividueller Eigenregulation, teils völlig konträr zu eventuellen Vergleichspersonen zu bieten. Daher ist im „Mittel“ über alle Personen möglicherweise kein Effekt zu dokumentieren, wenn man es in Studien so als Ergebnis erhalten will.

Trotzdem gibt es Effekte, die eigentlich vor Einführung des Mobilfunks hätten abgeklärt werden müssen. Hierzu zählen:

- Erhöhung der Stickoxid(NO)-Gaskonzentration der Ausatmungsluft durch Erhöhung der NO-Synthaseaktivität (Exspirationsluft) [1]
- Störung der Blut-Hirn-Schranke, messbar über Transthyretrin und Protein S100B [2]

- paradoxe Regulationsstörung in der Thermografie [1,3]

- ADHS-Syndrom bei neugeborenen Mäusen nach Exposition schwangerer Mäuse mit Mobilfunk [4]

- Zusammenhang Neurostressanalysen und Mobilfunk [5,6]

- SOD (Superoxiddismutase) ist ein elektromagnetisch empfindliches Enzym, welches im nitrosativen Stress eine übergeordnete Rolle spielt [7].

- NO-Gas zur Einleitung des programmierten Zelltods (Apoptose, im Gehirn unerwünscht)

- hohe NO-Spiegel, die mit Alzheimer in Verbindung gebracht werden. Die 


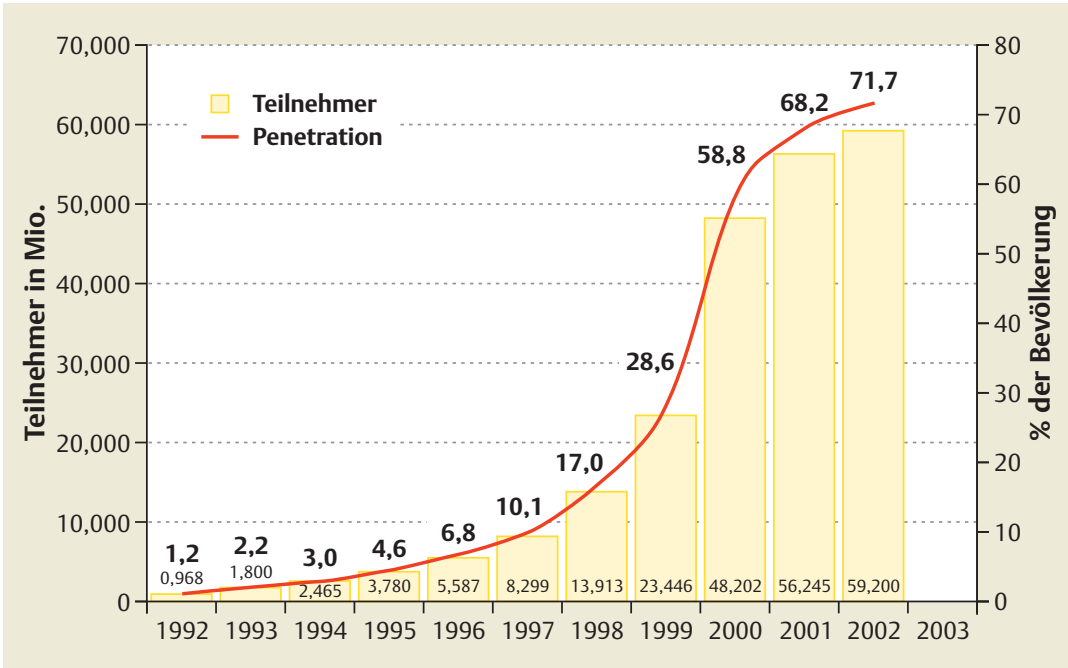

Abb. 1 Zunahme der Mobilfunkteilnehmer (Quelle: Bundesnetzagentur [14]).

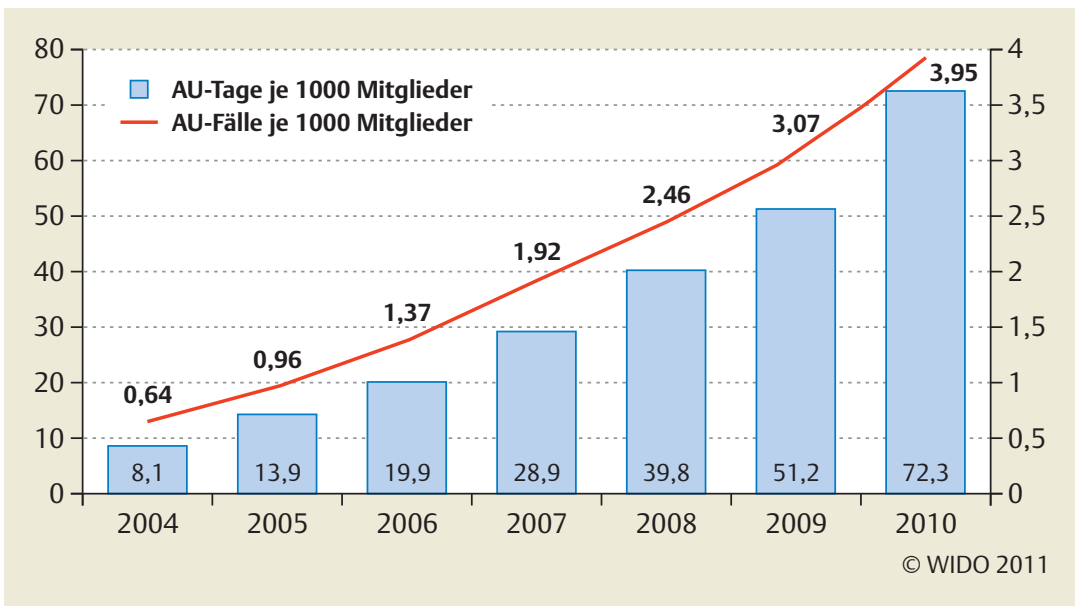

Abb. 2 Zunahme von Burn-out: Probleme mit Bezug auf Schwierigkeiten bei der Lebensbewältigung (Quelle: Wissenschaftliches Institut der AOK [15]).

alte Theorie von den $\beta$-AmyloidPlaques als Auslöser ist dagegen inzwischen verlassen worden, da dies ein sekundäres Phänomen ist [8-10].

Prof. Ulrich Warnke von der Universität des Saarlandes berichtet über die rasche nasale NO-Ausatmung unter relativ kurzer Exposition mit Mobilfunk [1]. Sehr einfach nachweisbar ist eine dauerhaft zu hohe NO-Belastung heute durch die Analyse der Abbauprodukte des NOStoffwechsels im Urin Citrullin, NitroPhenyl-Essigsäure und Methylmalonsäure.
— erhöht wesentlich die Insulinsensibilität

- senkt den Lungengefäßwiderstand und verhindert so pulmonalen Hochdruck

- moduliert die Genexpression in den verschiedenen Zelltypen und kann prinzipiell auch mit Zinkfinger-Transkriptionsfaktoren interagieren

- physiologischer Neurotransmitter im Gehirn für das Langzeitgedächtnis [11]

- Einleitung des programmierten Zelltods (wichtig bei Krebs)

\section{Nitrosativer Stress}

Bei „nitrosativem Stress“ handelt sich um eine das natürliche Maß übersteigende Produktion bzw. mangelnden Abbau des Moleküls NO, auch als NOX oder INOX (für induziertes NO-Gas) bezeichnet. Dieses Molekül muss im Körper in einem ausgewogenen Gleichgewicht vorliegen. Bei chronisch nitrosativem und oxidativem Stress ist es eine Frage der Zeit, wann irreversible Mitochondrien-Genschäden auftreten. Die mitochondriale DNA ist zehnfach empfindlicher gegen nitrosativen/oxidativen Stress verglichen mit der DNA im Zellkern.

Pall hat in seinem Buch „Explaining unexplained illnesses " auf den nitrosativen Stress als Pathomechanismus von vielen uns heute bekannten neurologischen Erkrankungen verwiesen [12]. Die Ignorierung dieser Erkenntnisse führt stattdessen zur Psychiatrisierung der Patienten, die beispielsweise an Fatigue oder MCS (Multipe ChemikalienSensibilität) leiden.

NO-Gas hat im physiologischen Bereich natürliche, wichtige Funktionen:

- Abwehr von Bakterien, Viren, Pilzen, bei der TH1-dominanten Immunreaktion

- unterbindet die Verklumpung von Blutplättchen

- verhindert, dass Entzündungszellen in die Gefäßwand einwandern

v sorgt für eine gute Sauerstoffversorgung des Gewebes

\section{Erkrankungen durch Funkstrahlung}

Schon sehr lange ist in der Wissenschaft das „Mikrowellensyndrom der Funkfrequenzkrankheit" eine medizinische Realität. Zu den Hauptsymptomen, die bereits vor 1932 veröffentlicht wurden, zählen: 


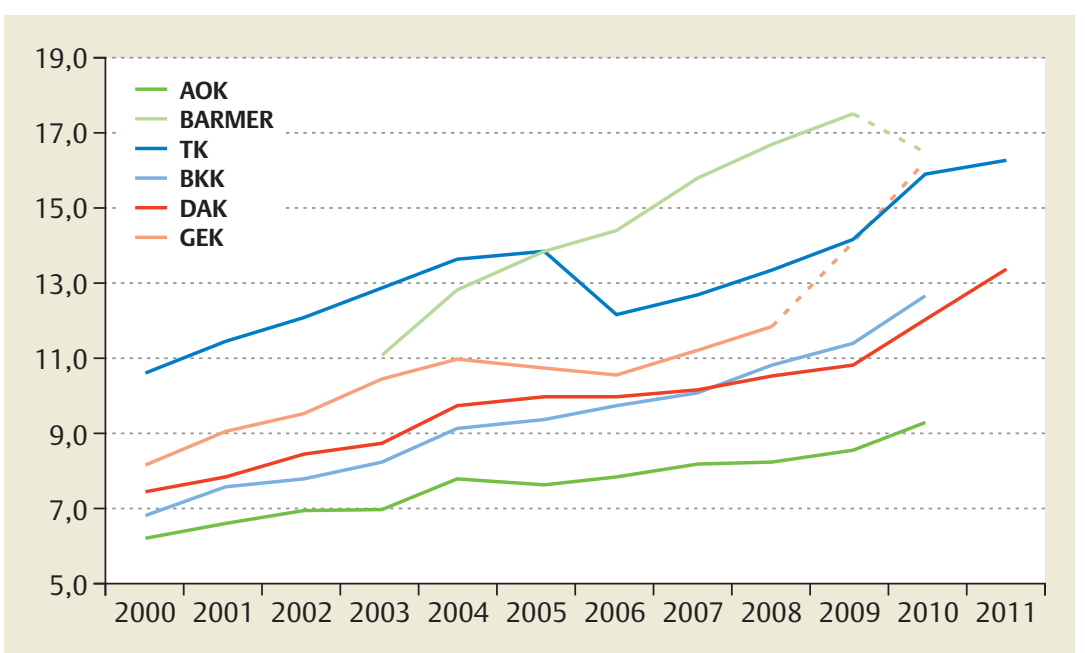

Abb. 3 Durch psychische Erkrankungen bedingte AU-Tage einzelner Krankenkassen (Quelle: Bundespsychotherapeutenkammer [16]).

- Erschöpfungssyndrom mit Müdigkeit, Reizbarkeit, Kopfschmerzen, Übelkeit und Appetitlosigkeit

- dystonisches kardiovaskuläres Syndrom: Herzrhythmusstörungen und arterielle Blutdruckstörungen

- dienzephalisches Syndrom mit Ermüdung, Schlaflosigkeit, Störungen der Sinne

Verglichen mit dem Jahr 2000 ist die Zahl der Störungen des zentralen Nervensystems unter 15-17-jährigen Jugendlichen um $85 \%$ gestiegen [13], die Anzahl der Personen mit Epilepsie oder epileptischen Erkrankungen hat um $36 \%$ zugenommen.

Interessanterweise wird sowohl von den Krankenkassen als auch von Therapeuten ein rasanter Anstieg von psychischen Erkrankungen registriert. Es korrelieren die Zunahme des Mobil-
Bedauerlicherweise ist die angesprochene Thematik der "technopathischen“ Einflussfaktoren - eine sinnvolle Wortschöpfung durch Dr. med. Peter Rothdach - inzwischen aus der medizinischen Wahrnehmung fast verschwunden. Wenngleich auch zahlreiche Möglichkeiten der orthomolekularen Therapie des nitrosativen Stresses existieren (siehe hierzu vorangegangene Ausgaben der OM), so sollte doch die Vermeidung von Auslösefaktoren Vorrang haben.

\section{( Literatur}

Die Literaturliste finden Sie im Internet unter: www.thieme-connect.de/ejournals

funks und der Anstieg psychischer Erkrankungen exakt - mit einigen Jahren Verzögerung ( $\rightarrow$ Abb. 1, 2 und 3).

\section{Fazit}

Die Korrelation zwischen Mobilfunk und psychischen Erkrankungen lässt sich nicht nur aus den epidemiologischen Daten, der Zunahme der Stickoxidexhalation unter Mobilfunkeinfluss und der Veränderung von NO-Markern im Urin ableiten. Unterstützung erhält die These auch durch Veröffentlichungen über Veränderungen der Blut-HirnSchranke sowie einer elektromagnetischen Sensibilität der SOD (Superoxiddismutase), einem Schlüsselenzym auch in der NO-Radikal-Genese und der Beteiligung von NO an Alzheimer. online: http://dx.doi.org/10.1055/s-0032-1328304

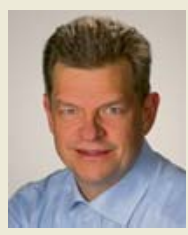
Facharzt für Allgemeinmedizin, Naturheilverfahren Katernberger Str. 76 42115 Wuppertal E-Mail: juergen@ aschoff-praxis.de

Jürgen Aschoff ist seit 1992 niedergelassen als Arzt für Allgemeinmedizin und Naturheilverfahren. Seit 1998 arbeitet er als Dozent der DGNS (Deutsche Gesellschaft für Naturheilkunde und energetische Schmerztherapie e. V.). Eigene Forschung zu Frequenzanalytik und Therapie bei Patienten, Studie über Veränderungen des Immunstatus vor und nach Frequenztherapie, Fortführung des vom Vater entwickelten Elektromagnetischen Bluttests zur Ursachenerkennung der Krankheitsprozesse.
Jürgen Aschoff 